\title{
Site of Directly Observed Therapy
}

National Cancer Institute

\section{Source}

National Cancer Institute. Site of Directly Observed Therapy. NCI Thesaurus. Code C102710.

The physical location at which the medication or therapy was observed, by the healthcare provider, as being taken by the subject. 